\title{
Factoring in scheduled repairs of generating units when assessing the resource adequacy of electric power systems
}

\author{
Dmitry Krupenev ${ }^{1, *}$, Denis Boyarkin ${ }^{1}$, and Dmitrii Iakubovskii ${ }^{1}$ \\ ${ }^{1}$ Melentiev Energy Systems Institute of Siberian Branch of the Russian Academy of Sciences, 664033, Irkutsk, Lermontov 130 st..
}

\begin{abstract}
This study presents the algorithms for modeling of scheduled repairs of generating equipment when assessing the resource adequacy of electric power systems by the Monte Carlo method that relies on random events. We present an analysis of the algorithms adopted in the available software and computer systems used to assess the resource adequacy and highlight their shortcomings. We propose an algorithm for modeling of scheduled repairs of generating units that does away with the flaws of the algorithms adopted elsewhere. In the final part of the article, we present the findings of numerical experiments that test the performance of the algorithms. Keywords: resource adequacy, electric power system, scheduled repairs, generating unit, Monte Carlo method.
\end{abstract}

\section{Introduction}

Assessment of the reliability of electric power systems (EPS) is an integral procedure for solving many problems of control over the operation and development of the EPS. When planning the development of the EPS, it is necessary to assess the balance reliability to substantiate the regulatory reserve requirements to be met by generating capacity, the structure, and throughput capacity of the power grid. The problem of assessing the EPS resource adequacy can be stated as follows: For given annual hourly profiles of power consumption, the composition, technical and reliability parameters of generating equipment within the reliability zones, the composition, technical and reliability parameters of power lines in the inter-zone connections to determine the resource adequacy for the reliability zones and for the entire system for a one-year period. Reliability parameters of generating equipment should include scheduled repairs.

In the electric power industry, scheduled repairs are a mandatory procedure. There is a regulation on the timing and amount of repairs of power facilities. For most types of the power equipment operated in Russia, this regulation and the rules for taking generating equipment out of service for repairs are presented in [1-3]. The issues of scheduled repairs of nuclear power plants and some other types of power equipment were not addressed there.

Ultimately, reliability of power supply to consumers and reliability of power plants depend on timely carrying out of scheduled repairs. The functional relation is manifested in the increase in the fault rate of power equipment. Repairs of energy equipment require certain costs, but it is known that the damage in case of failure of the unrepaired equipment exceeds the costs.

Since the resource adequacy assessment is carried out for prospective schemes of EPS development, the plans for repairs of generating equipment are oftentimes unknown. Under such circumstances, scheduled repairs of generating equipment are factored in based on custom-built algorithms that simulate real rules for working out repair schedules. An algorithm for modelling of repair schedules should most accurately reflect the actual conditions of repairs of generating equipment. This study covers the algorithms for modelling scheduled repairs of generating equipment employed for balance reliability assessment in the available software and computer systems (SCS), identifies their shortcomings, and proposes a new algorithm that improves the process of modelling of scheduled repairs of generating equipment as part of the assessment of resource adequacy of the EPS.

\section{Algorithms for modelling of scheduled repairs}

The methodology behind assessing the resource adequacy of the EPS that relies on the Monte Carlo method is made up of the following three main steps [46]:

1. The step of generating random states of the EPS.

2. The step of minimizing the power shortage under random states of the EPS.

\footnotetext{
Corresponding author: krupenev@,isem.irk.ru
} 
3. The step of calculating the resource adequacy indicators of the EPS.

At the first step, alongside other calculation procedures, scheduled repairs of generating units are factored in. At the same time, the following aspects of the operation of the EPS should be taken into consideration as well:

- Special considerations for establishing requirements to be complied with by scheduled repairs of NPP, CCGT and GTU equipment;

- Scheduled repairs of generating equipment that operates in the heat and power generation mode outside the heating season;

- Scheduled repairs of hydropower plant (HPP) generating equipment outside the flood season;

- Planned repairs of generating equipment during periods of the seasonal decrease in consumption, taking into account the peculiarities of regional power systems characterized by summer peak power consumption;

- Compatibility with repairs of power lines, as well as other generating and power grid equipment;

- Amount of unscheduled and emergency repairs of generating equipment.

There are several types of scheduled repairs [1]: major repairs, medium repairs, and running repairs. All of them are defined by the scope of work to be performed and the timing of carrying out such scopes of work.

When assessing the resource adequacy of prospective EPS schemes, there may be several options with the information available on scheduled repairs of generating equipment. In case of long-term planning of the power system operation, plans for scheduled repairs of generating equipment are known up to 1 year ahead; these plans are developed by the System Operator when processing applications of generating companies. In such case, one should take generating units out of service as per these plans when assessing resource adequacy. For the period of planning of the operation and development of the EPS up to 3 years ahead, the quantities of taking generating units out of service for scheduled repairs may be known, but there is no known order and sequence of taking them down. For prospective schemes of EPS development for periods exceeding 3 years, the amount of planned repairs and the order of taking generating equipment out of service are not known, and when assessing the resource adequacy, which is usually carried out for a one-year period, it is assumed that the amount of scheduled repairs of generating units is equal to the average amount of scheduled repairs for each unit over its repair cycle, which is presented in [1]. Let us consider various options available for modelling of scheduled repairs of generating units when assessing the resource adequacy of the EPS.

\subsection{Factoring in scheduled repairs of generating units based on statistical data}

The easiest way to factor in scheduled repairs of generating units when assessing the balance reliability of the EPS is to factor them based on their statistical data of an earlier period. The algorithm behind this process is as follows:

1. Determination of the generation capacity within the reliability zones that was taken out of service for scheduled repairs as averaged over the past period:

$$
\begin{gathered}
x_{\text {avg }, s c h, i, \chi}=\frac{\sum_{n=1}^{N} x_{s c h, i, \chi}, n}{N}, \\
i=1, \ldots, I, \chi=1, \ldots, 12
\end{gathered}
$$

where: $x_{s c h, i, \chi, n}$ is the actual average amount of planned repairs of generating units within the reliability zones in month $\chi$ of year $n, \mathrm{MW}$; where $N$ is the number of years for which statistical data are used; $I$ is the number of reliability zones.

2. Determination of the operating generating capacity within the reliability zones in the design states of the EPS given scheduled repairs of generating units:

$$
\begin{gathered}
x_{o p, i}=x_{a v, i}-x_{e m, i}-x_{a v, s c h, i, \chi} \\
i=1, \ldots, I, \quad \chi=1, \ldots, 12
\end{gathered}
$$

where: $x_{a v, i}$ is available capacity of generating units within the reliability zones, MW; $x_{e m, i}$ is generating capacity within the reliability zones taken out of service for emergency repairs, MW.

The above approach in not free of the following shortcomings:

1. Lack and unavailability of information on the statistical data on scheduled repairs of generating units.

2. Changing conditions for the operation of EPS in prospective schemes, including the commissioning and decommissioning of generating equipment.

3. Duplication of generating units when factoring in emergency and scheduled repairs.

\subsection{Factoring in scheduled repairs of generating units into the load profile}

This algorithm has been implemented in the Yantar software and computer system (SCS) [4] and other SCSs. Major and medium repairs are factored in by means of a dip in the load profile for monthly peaks of reliability zones. The algorithm behind such factoring in consists of the following steps:

1. Determination of the amount of medium and major repairs of generating equipment within the reliability zones averaged over a year as measured in MW per day:

$$
V_{a m r, i}=\sum_{h=1}^{H} x_{h, i} t_{a m r, h, i}, i=1, \ldots, I
$$

where $x_{h, i}$ is the available capacity of generating unit $h$ in reliability zone $i, \mathrm{MW} ; \mathrm{H}$ is the number of generating units in reliability zone $i$; $t_{a m g, h, i}$ is the average annual time to carry out medium and major repairs of generating unit h in reliability zone $i$ per year, days.

2. Ordering (ranking) of the monthly peak power consumption profile from the minimum to the maximum 
value in each of the reliability zones. When ranking, the information on which calendar month a given value belongs to is retained.

3. Determination of the amount of medium and major repairs each month. For this purpose, a loop is introduced to fill in the dip of the ranked monthly peak load profile starting from the minimum load on, proceeding in ascending order until $V_{a m r, i}$ is fully included in the annual load profile of the reliability zone. As a result, for each $\chi^{\text {th }}$ month the value of the "amount" of medium and major repairs will be obtained for the reliability zones $V_{a m r, i, x}$.

4. Determination of the value of generating capacity taken out for medium and major repairs within the reliability zones:

$$
x_{a m r, i, \chi}=\frac{V_{a m r, i, \chi}}{N}, i=1, \ldots, I, \chi=1, \ldots, 12,
$$

where: $N$ is the number of days in each month.

5. Determination of the values of the amount of running repairs of generating equipment within the reliability zones averaged over a year as measured by MW a day as per the following formula:

$$
V_{r r, i}=\sum_{h=1}^{H} x_{h, i} t_{r r, h, i}, i=1, \ldots, I
$$

where $t_{r r, h, i}$ is the average annual time for running repairs of generating unit $h$ in reliability zone $i$ per year, days.

6. Determination of the value of the generating capacity taken out for running repairs in the reliability zones:

$$
x_{r r, i}=\frac{V_{r r, i}}{365}, i=1, \ldots, I .
$$

7. Generation of the monthly peak values profile with scheduled repairs of generating equipment within the reliability zones factored in:

$$
\begin{gathered}
\bar{y}_{s c h, i, \chi}=y_{i, \chi}+x_{a m r, i, \chi}+x_{r r, i} \\
i=1, \ldots, I, \chi=1, \ldots, 12
\end{gathered}
$$

where $\bar{y}_{i, \chi}$ is the peak load in reliability zone in month $\chi, \mathrm{MW} ; x_{a m r, i, \chi}$ is the amount of generating capacity taken out of service for major and medium repairs in reliability zone $i$ in month $\chi, \mathrm{MW} ; x_{r r, i}$ is the amount of generating capacity taken out of service for running repairs in reliability zone $i, \mathrm{MW}$.

Figure 1 shows the process of generating the load profile with scheduled repairs of generating equipment within the reliability zones factored in.

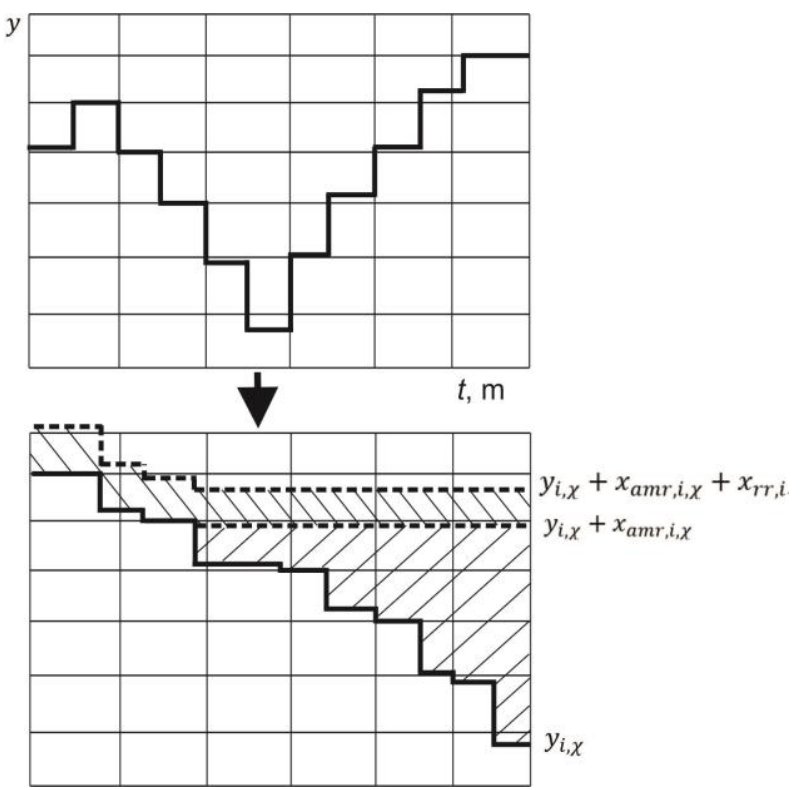

Fig. 1. The process of generating the load profile with scheduled repairs of generating equipment within the reliability zones factored in.

The main drawbacks of this approach to factoring in scheduled repairs are as follows:

1. Duplication of generating units when factoring in emergency and scheduled repairs.

2. "Smoothing out" the timetable of scheduled repairs within the reliability zones with a small number of generating units and / or a minor dip in the load profile. If the profile of monthly load peaks is "smooth" and is free of a pronounced dip, then within the reliability zones with a small number of generating units, the amount of medium and major repairs will be distributed over several months, which distorts the actual situation in the EPS, according to which each generating unit is under scheduled repairs for a certain number of days of a month.

\subsection{Factoring in scheduled repairs of generating units when determining the operating capacity of reliability zones}

This approach is based on the combination of the two above presented approaches to factoring in scheduled repairs of generating equipment when assessing the EPS resource adequacy. The approach consists of the following steps:

1. Determination of the amount of scheduled repairs in each month of the reference year according to the methodology presented as part of the previous approach (items 1 to 6 ).

2. Determination of the values of generating capacity within reliability zones that is taken out of service for scheduled repairs each month:

$$
\begin{aligned}
& x_{s c r, i, \chi}=x_{a m r, i, \chi}+x_{r r, i} \\
& i=1, \ldots, I, \chi=1, \ldots, 12 .
\end{aligned}
$$


3. Determination of the operating generating capacity within the reliability zones in design states of the EPS given scheduled repairs of generating units:

$$
\begin{gathered}
x_{o p e, i}=x_{a v, i}-x_{a c c, i}-x_{s c h, i, \chi} \\
i=1, \ldots, I, \quad \chi=1, \ldots, 12
\end{gathered}
$$

The approach has the same flaws as the previous one.

\subsection{Factoring in scheduled repairs of generating units in the process of generating design states of the EPS}

In this algorithm, unlike with those presented earlier, scheduled repairs can be incorporated not only into the load profile of the reliability zone, but also into a larger interconnection, for example, in the case of the Unified Power System of Russia the latter can be interconnected power systems (IPS). In this case, there will be a dip in the load profile with a probability higher than the one obtained when considering an individual reliability zone. Where an isolated system or a system with loose external links is considered, and it has a relatively "smooth" profile of monthly peak loads and a small number of generating units, individual rules for assigning generating equipment for repairs should be applied to this system. One more feature of this algorithm is that scheduled repairs are dealt with as a part of the same computational procedure as emergency repairs which rules out a possibility of their duplication. The algorithm behind this approach consists of the following steps:

1. The first six steps of this algorithm are similar to the first six steps of the second algorithm presented above, with the only difference being that instead of reliability zones it is the parts of the power system that are considered: they may include multiple reliability zones and are generated by experts. As a result, expression (8) determines the values of the generating capacity taken out of service for scheduled repairs in individual parts of power systems.

2. Determination of the relative amount of scheduled repairs within each interconnected power system each month:

$$
k_{s c h, i, \chi}=\frac{x_{s c h, i, \chi}}{x_{a v, i}} i=1, \ldots, I, \chi=1, \ldots, 12
$$

3. Determination of the share of the time each generating unit is under scheduled repairs relative to the total downtime due to scheduled repairs of all generating units in an individual part of the energy system $i$ :

$$
t_{a v, h, i}^{\prime}=\frac{t_{a v, h, i}}{\sum_{h=1}^{H} t_{a v, h, i}}, i=1, \ldots, I,
$$

where: $t_{s c h, h, i}$ - average annual time for scheduled repairs of generating unit $h$ in power system's part $i$ per year; $H$ is the number of generating units.

4. Determination of coefficients used to adjust the availability of generating units for scheduled repairs: $k_{a d j, s c h, h, i}=t_{s c h, h, i}^{\prime} \cdot H, h=1, \ldots, H, i=1, \ldots, I$.

5. Determination of the relative amount of scheduled repairs of generating units within each interconnected power system each month:

$$
\begin{gathered}
k_{a d j, h, i, \chi}=k_{s c h, i, \chi} \cdot k_{a j d, s c h, h, i}, \\
h=1, \ldots, H, i=1, \ldots, I, \chi=1, \ldots, 12 .
\end{gathered}
$$

Coefficient $k_{s c h, h, i_{1},}$ is used as an additional parameter for playing out the states of the generating equipment, it is to be summed up with its fault rate.

\section{Experimental studies of the algorithms under consideration}

Experimental studies of the algorithms were carried out based on the three-zone model of the EPS presented in Figure 2.

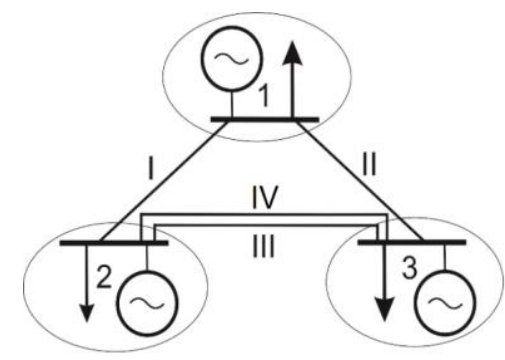

Fig. 2. Schematic diagram of the study EPS.

Table 1 presents the main characteristics of the reliability zones of the study EPS. Reliability zones No. 1 and 2 have a small number of generating units 7 and 6 , respectively, while reliability zone No. 3 have 45 generating units to it. The available capacity of the EPS under consideration is $6,050 \mathrm{MW}$, the peak load of the power system is $5,800 \mathrm{MW}$, and the capacity margin is $14 \%$. Table 2 presents the main characteristics of interzone links. Figure 3 presents profiles of monthly peak loads of reliability zones. As noted above, one of the main challenges when factoring in scheduled repairs by means of the dip of the load profile of the reliability zone

\begin{tabular}{|c|c|c|c|c|c|c|c|}
\hline \multirow{2}{*}{$\begin{array}{c}\text { Relia } \\
\text { bility } \\
\text { zone } \\
\text { No. }\end{array}$} & \multirow{2}{*}{$\begin{array}{l}\text { Avail } \\
\text { able } \\
\text { capaci } \\
\text { ty, } \\
\text { MW }\end{array}$} & \multirow{2}{*}{$\begin{array}{c}\text { Nu } \\
\text { mb } \\
\text { er } \\
\text { of } \\
\text { uni } \\
\text { ts }\end{array}$} & \multirow{2}{*}{$\begin{array}{c}\text { Gener } \\
\text { ating } \\
\text { unit } \\
\text { fault } \\
\text { rate }\end{array}$} & \multicolumn{3}{|c|}{$\begin{array}{c}\text { Average } \\
\text { amount of } \\
\text { repairs per } \\
\text { year, days }\end{array}$} & \multirow{2}{*}{$\begin{array}{l}\text { Maxi } \\
\text { mum } \\
\text { load, } \\
\text { MW }\end{array}$} \\
\hline & & & & $\begin{array}{l}\text { M } \\
\text { aj }\end{array}$ & $\begin{array}{c}\mathrm{Me} \\
\mathrm{d}\end{array}$ & Run & \\
\hline \multirow{2}{*}{1} & 50 & 5 & 0.05 & 5 & & 3.6 & \multirow{2}{*}{400} \\
\hline & 100 & 2 & 0.05 & 8 & 3.2 & 4.8 & \\
\hline
\end{tabular}
is its "smooth" form. In this particular case, the profiles of monthly peaks of the second and third reliability zones are "smooth" relative to the first reliability zone.

Table 1. Characteristics of reliability zones of the study EPS. 


\begin{tabular}{|c|c|c|c|c|c|c|c|}
\hline \multirow{2}{*}{2} & 50 & 5 & 0.05 & 5 & & 3.6 & \multirow{2}{*}{400} \\
\cline { 2 - 7 } & 100 & 1 & 0.05 & 8 & 3.2 & 4.8 & \\
\hline \multirow{3}{*}{3} & 50 & 15 & 0.05 & 5 & & 3.6 & \\
\cline { 2 - 6 } & 100 & 15 & 0.05 & 8 & 3.2 & 4.8 & \multirow{2}{*}{4500} \\
\cline { 2 - 6 } & 200 & 15 & 0.05 & $\begin{array}{c}12 \\
.3\end{array}$ & $\begin{array}{c}6.2 \\
5\end{array}$ & $\begin{array}{c}14 . \\
5\end{array}$ & \\
\hline
\end{tabular}

Note: Maj - major repairs; Med - medium repairs; Run running repairs.

Table 2. Description of inter-zone links of the study EPS.

\begin{tabular}{|c|c|c|c|c|}
\hline Link & $\begin{array}{c}\text { Capacit } \\
\text { y limit, } \\
\text { MW }\end{array}$ & $\begin{array}{c}\text { Length, } \\
\mathbf{~ k m}\end{array}$ & $\begin{array}{c}\text { Fault } \\
\text { rate, } \\
\text { unit } \\
\text { fraction } \\
\text { /100km }\end{array}$ & $\begin{array}{c}\text { Specific } \\
\text { loss } \\
\text { factor }\end{array}$ \\
\hline $1-2$ & 135 & 400 & 0.001 & 0.0001 \\
\hline $1-3$ & 135 & 400 & 0.001 & 0.0001 \\
\hline $2-3$ & 135 & 400 & 0.001 & 0.0001 \\
\hline $2-3$ & 135 & 400 & 0.001 & 0.0001 \\
\hline
\end{tabular}

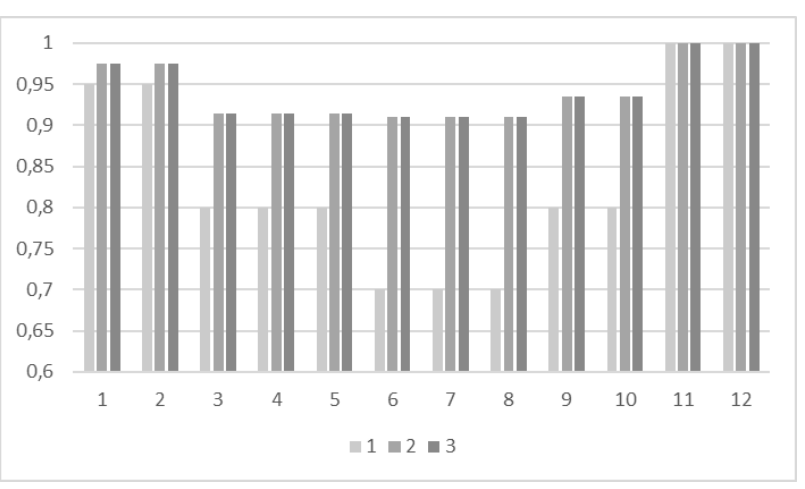

Fig. 3. Schedule of monthly maximums of the load of the reliability zones, the investigated EPS, p.u. (relative to annual maximum load).

Table 3 presents the results of the resource adequacy assessment by the second and fourth algorithms of modeling of scheduled repairs of generating units.
Table 3. Probability values of shortage-free operation when using various algorithms of modeling of scheduled repairs of generating units

\begin{tabular}{|c|c|c|}
\hline \multirow{2}{*}{$\begin{array}{c}\text { Reliability } \\
\text { zone } \\
\text { number }\end{array}$} & \multicolumn{2}{|c|}{$\begin{array}{c}\text { Probability of shortage-free operation } \\
\text { mm }\end{array}$} \\
\cline { 2 - 3 } & Algorithm 2 & Algorithm 4 \\
\hline 1 & 0.9991 & 0.9958 \\
\hline 2 & 0.9965 & 0.9833 \\
\hline 3 & 0.9963 & 0.9756 \\
\hline System & 0.9951 & 0.9709 \\
\hline
\end{tabular}

As can be seen from the findings presented in Table 3 , the algorithm for modeling of scheduled repairs of generating units affects the probability of shortage-free operation of the EPS. In the case of algorithm No. 4 that we have developed, the probability values for shortagefree operation are lower than those in the case of algorithm No. 2. This is due to the above indicated shortcomings of algorithm No. 2, although algorithm No. 4 can still be improved upon as well in order to provide more satisfactory modeling of scheduled repairs of generating units when assessing the EPS resource adequacy. The study of algorithms was performed based on a test scheme that has zones of reliability with a small number of generating units and a "smooth" profile of monthly peak loads. This is what makes the above difference in the results. The biggest difference in the probability of shortage-free operation is in Zones 2 and 3 , as they have a "smoother" profile of monthly peak loads (see Figure 3). When examining the resource adequacy of real-life EPS, similar situations with the load profile and the number of generating units within the reliability zones occur less frequently, so the difference in resource adequacy indicators will be less pronounced.

\section{Conclusion}

Dedicated software and computer systems are used to assess the EPS resource adequacy. In these systems, one of the procedures is to factor in scheduled repairs of generating units. The efficacy of an algorithm used for scheduled repairs determines the accuracy of the resource adequacy indicators obtained. On the basis of the analysis of the employed algorithms of factoring in scheduled repairs when assessing the resource adequacy relying on the Monte Carlo method, it is possible to draw a conclusion that all the algorithms have their inherent assumptions affecting the accuracy of the reliability indicators. This study proposes a new algorithm that makes for more accurate assessment of the resource adequacy of electric power systems. In the course of experimental studies, the differences that arise 
when using the algorithm advocated by the authors and the algorithm used in existing software and computer systems were highlighted.

The research was carried out under State Assignment 17.5.3 (reg. no, AAAAA-A17-117030310450-3).

\section{References}

1. Rules for managing the maintenance and repairs of electric power industry facilities. Order of the Ministry of Energy of the Russian Federation dated October 25, 2017, No. 1013. (in Russian)

2. GOST R 57285-2016. Unified electric power system and isolated power systems. Electric power systems. Operations and dispatch control. The procedure of drawing up final reports on the possibility of decommissioning of power plant generating equipment related to dispatching facilities. Standards and requirements. (in Russian)

3. On taking electric power facilities out of service for repairs and their decommissioning. Decree of the Government of the Russian Federation, dated July 26, 2007, N 484. (in Russian)

4. Kovalev G.F., Lebedeva L.M. Reliability of Power Systems. Springer. 2019. 237 p.

5. Billinton R., Li W. Reliability Assessment of Electric Power Systems Using Monte Carlo Methods. Springer. 1994. $361 \mathrm{p}$.

6. Boyarkin D.A., Krupenev D.S., Iakubovskii D.V. Machine learning in electric power systems adequacy assessment using Monte-Carlo method. Bulletin of the South Ural State University, Series: Mathematical Modelling, Programming and Computer Software. Vol.11. No.4. 2018. P.146-153. 